\section{The Psychological Effects of COVID-19 Pandemic Related Lockdown in Children}

In India, varying degrees of lockdown due to coronavirus disease 2019 (COVID-19) pandemic have been enforced in most states since late March, 2020, which has extended for more than four months now. Schools across India have closed and restrictions on public movement is in effect. Most children are forced to stay indoors because of this lockdown, which has significantly disrupted their routine and reduced social contact.

This prolonged indoor stay is likely to affect their psychological well-being in many ways. Restriction of movement, inability to indulge in physical outdoor sports activities, reduced social contact with peer group, monotonous daily routine and difficulty in being engaged can have a negative bearing on the child. The childhood psychological reactions to COVID-19 pandemic can be broadly classified into internalizing problems like anxiety, depression, withdrawn state and somatic complaints and externalizing problems like irritable states, aggression, disruptive and rule breaking behavioral responses. An Italian study done among children during COVID lockdown reported an increase in externalizing tendencies like irritability, intolerance to rules, whims and excessive demands [1]. A similar Spanish study showed increase in nervousness, worry, feeling of loneliness, boredom and anger which includes both internalizing and externalizing tendencies [2]. Lockdown also impaired the quality of sleep and sleep stabilization in children [3]. Quarantined Indian children were found to experience greater psychological distress like worry, helplessness and fear [4]. On the other hand, reduction in academic pressure and more time spent with family may also contribute to reduction in stress.
Our understanding about the psychological effects of COVID-19 lockdown in children is still evolving. Most of the available data are based on unvalidated, ad hoc questionnairebased studies with poor external validity and have to be interpreted cautiously. More studies on this aspect are needed in order to understand and prevent psychological problems in children

ThirunaVukKarasu Arun Babu ${ }^{*}$ and JAIGANESH SElVAPANDIYAN ${ }^{2}$ From Departments of ${ }^{1}$ Pediatrics and ${ }^{2}$ Psychiatry, All India Institute of Medical Sciences (AIIMS), Mangalagiri, Andhra Pradesh, India

*babuarun@yahoo.com

\section{REFERENCES}

1. Pisano L, Galimi D, Cerniglia L. A qualitative report on exploratory data on the possible emotional/behavioral correlates of Covid-19 lockdown in 4-10 years children in Italy. [Preprint]. PsyArXiv. 2020. Available from: https:// psyarxiv.com/stwbn.

2. Idoiaga Mondragon N, Berasategi Sancho N, Dosil Santamaria M, Eiguren Munitis A. Struggling to breathe: A qualitative study of children's wellbeing during lockdown in Spain. Psychol Health. 2020; 1-16. Available from: https:// www.tandfonline.com/doi/full/10.1080/ 08870446.2020.1804570. [Epub ahead of print].

3. Dellagiulia A, Lionetti F, Fasolo M, Verderame C, Sperati A, Alessandri G. Early impact of COVID-19 lockdown on children's sleep: A four-week longitudinal study. J Clin Sleep Med. 2020; jcsm-8648. Available from: https:// jcsm.aasm.org/doi/10.5664/jcsm.8648. Accessed July 6, 2020. [Epub ahead of print].

4. Saurabh K, Ranjan S. Compliance and psychological impact of quarantine in children and adolescents due to COVID-19 pandemic. Indian J Pediatr. 2020;87:532-6.

\section{Do All Children With Autoimmune Encephalitis Need Aggressive Immunotherapy?}

Autoimmune encephalitis is increasingly being recognized in children in India, and the recent review was therefore timely [1]. Many centers have reported that autoimmune encephalitis as a group is more common than encephalitis caused by any single etiogical agent like virus/bacteria [2]. They usually have an acute to subacute poly-symptomatic presentation [3].

Prompt immunotherapy in time gives good neurological outcomes in most of them. As antibody testing results takes few days, many clinico-laboratory criteria have been proposed for diagnosis of early possible/probable and seronegative autoimmune encephalitis so that treatment can be started pending the results [4]. This puts the pediatrician under pressure to start immunomodulation after common infectious causes are ruled out. Which drugs to use for immunomodulation is not clear from the literature. Though many authors and guidelines advocate use of methylprednisolone pulse therapy (MP) with intravenous immunoglobulin (IVIG) as the initial modality of treatment, it is not clear whether they should be used simultaneously or sequentially [3]. If sequentially, after how many days of methylprednisolone therapy should IVIG be used is also not clear. Whether IVIG should be used in all or on the basis of clinical severity or response to MP is also unclear.

Like Guillain-Barre syndrome and many other diseases, there is a spectrum of severity of autoimmune encephalitis and all children may not need aggressive treatment with both MP and IVIG. While milder ones may be self-limiting, the mild to moderately severe ones may need just 2-3 cycles of MP and the severe ones may need more than one agent and chronic immunomodulation for longer duration. Most of the milder forms of autoimmune encephalitis may not reach the tertiary 


\section{CORRESPONDENCE}

care centers also and these cases may be missed from the series of tertiary care centers.

While working in a medical college situated in a district place, we have managed six cases of anti-NMDAR autoimmune encephalitis (antibody confirmed) with poly-symptomatic presentation in the last four years. Four of them responded to just 3-6 monthly cycles of MP while only two (presented to us more than 4 weeks after onset) had to be given either IVIG or rituximab after methylprednisolone because of lack of response to MP in one week. Most of them could not afford IVIG/ rituximab, and received only MP. They showed good clinical response. None of the children who received MP have had any relapse (duration of follow-up: 6 month-4 years).

We want to suggest that all children with anti-NMDAR antibody encephalitis do not need aggressive immunomodulation with MP, IVIG and other agents. Many of them may just respond to 3-6 doses of MP alone. However more studies including milder cases from peripheral centers are needed to generate robust evidence on this aspect.

MAHESH KAMATE* AND BR RITESH Division of Pediatric Neurology, Department of Pediatrics, KAHER's Jawaharlal Nehru Medical College Belagavi, Karnataka, India. *drmaheshkamate@gmail.com

\section{REFERENCES}

1. Garg D, Mohammad SS, Sharma S. Autoimmune encephalitis in children: An update. Indian Pediatr. 2020;57:662-70.

2. Gable MS, Sheriff H, Dalmau J, Tilley DH, Glaser CA. The frequency of autoimmune N-methyl-D-aspartate receptor encephalitis surpasses that of individual viral etiologies in young individuals enrolled in the California Encephalitis Project. Clin Infect Dis. 2012;54:899-904.

3. Zuliani L, Nosadini M, Gastaldi M, et al. Management of antibody-mediated autoimmune encephalitis in adults and children: literature review and consensus-based practical recommendations. Neurol Sci. 2019;40:2017-30.

4. Cellucci T, Van Mater H, Graus F, et al. Clinical approach to the diagnosis of autoimmune encephalitis in the pediatric patient. Neurol Neuroimmunol Neuroinflam. 2020;7.

\section{AUTHORS'REPLY}

We thank the readers for their interest in our publication [1]. Several expert recommendations for treatment for autoimmune encephalitis are available but there is no uniform standardized approach to therapy. However, the degree of aggressiveness of therapy needed may often be clinically guided by certain prognostic factors in an individual patient. Additionally, second-line agents such as rituximab or cyclophosphamide are used when first-line agents (steroids, intravenous immunoglobulin or plasmapheresis) fail. In a cohort of 577 patients with anti-NMDAR encephalitis, of whom 211 were children, 94\% underwent first-line therapy/tumor removal, and $53 \%$ improved within 4 weeks. Most patients improved with immunotherapy, with $81 \%$ living independently two years after the diagnosis [2]. In this cohort, predictors of favorable outcome (including in 177 of 211 children) were early initiation of treatment and lack of intensive care unit (ICU) admission. Hence, authorities recommend escalation to second-line therapy if there is lack of significant improvement on first-line therapy in 10-14 days in anti-NMDAR encephalitis, especially among patients admitted to the ICU [3]. However, this brisk escalation may not be warranted for other autoimmune encephalitis and clinicians may wait longer before introducing second-line therapy [4].

While steroids are definitively therapeutic in this condition, there are certain issues with their use. Often, it is difficult to differentiate infectious causes of encephalitis from autoimmune encephalitis, and steroid initiation may be delayed. Additionally, the immunological effects of steroids are much more on T-cells compared to B-cells, and since autoimmune encephalitis is antibody-mediated largely, whether steroids alone would be as effective in all cases of autoimmune encephalitis is uncertain [5].

However, as the authors note, there are no clear guidelines on how frequently to repeat steroid dosing and the dosing interval is usually dictated by severity of the disease and the response of the child to therapy, including relapses. Indeed, all children may not warrant aggressive immunotherapy and it is better to individualize treatment rather than the 'one-size-fitsall' approach.

\section{Divyani Garg ${ }^{1}$ and Suvasini Sharma ${ }^{2 *}$ \\ ${ }^{1}$ Departments of Neurology and \\ ${ }^{2}$ Pediatrics (Neurology Division), \\ Lady Hardinge Medical College, New Delhi, India. *sharma.suvasini@gmail.com}

\section{REFERENCES}

1. Garg D, Mohammad SS, Sharma S. Autoimmune encephalitis in children: An update. Indian Pediatr. 2020;57:662-70.

2. Titulaer MJ, McCracken L, Gabilondo I, et al. Treatment and prognostic factors for long-term outcome in patients with anti-NMDA receptor encephalitis: An observational cohort study. Lancet Neurol. 2013;12:157-65.

3. Dalmau J, Lancaster E, Martinez-Hernandez E, Rosenfeld MR, Balice-Gordon R. Clinical experience and laboratory investigations in patients with anti-NMDAR encephalitis. Lancet Neurol. 2011;10:63-74.

4. Bien CG, Bien CI. Autoimmune encephalitis in children and adolescents. Neurol Res Pract. 2020;2:4.

5. Shin Y-W, Lee S-T, Park K-I, et al. Treatment strategies for autoimmune encephalitis. Ther Adv Neurol Disord. 2018;11:1756285617722347. 